\title{
A study on Effects of Axle Misalignment of a Vehicle with Independently Rotating Wheels*
}

\author{
Katsuya TANIFUJI**, Hideaki KOBAYASHI** and Hitoshi SOMA** \\ ${ }^{* *}$ Department of Mechanical and Production Engineering, Niigata University, \\ 8050 Ikarashi 2-no-cho, Nishi-ku, Niigata-city, Niigata, Japan \\ E-mail: tanifuj@eng.niigata-u.ac.jp
}

\begin{abstract}
This paper is concerned with the effects of the axle misalignment of a truck with independently rotating wheels (IRWs) on the wheel lateral force and flange wear. Since a truck with IRWs lacks self-steering ability, it is of concern that the wheel flange remains in contact with the rail owing to the effect of axle misalignment in parallelity, resulting in rapid wear of the flange upon continuous contact with the rail. Numerical simulations are carried out using an analytical vehicle model with axle misalignment. The obtained results are as follows. Even if misalignment is small, a vehicle with IRWs tends to run under the flange contact condition, and thus a continuous lateral force acts between the flange and rail. A vehicle with IRWs that has axle misalignment only in the lateral direction does not tend to run with continuous flange contact. In the range of misalignment in parallelity considered in this paper, the wheel lateral force increases with increasing misalignment, and the wheel lateral force acting on the IRWs increases more rapidly than that acting on the solid wheelset. The wheel lateral force generated by misalignment is equivalent to that generated during the negotiation of a curved track with a certain radius, which causes the attack angle to correspond to the angle of misalignment.
\end{abstract}

Key words: Railway, Simulation, Modelling, IRW, Axle Misalignment, Wheel Lateral Force

\section{Introduction}

Generally, a solid wheelset (SW), in which two wheels are fixed on a common axle, is used as the wheelset in railway vehicles. On the other hand, vehicles with independently rotating wheels (IRWs), which rotate on the left and right ends of an irrotational axle through bearings, have been developed. IRWs were employed in the development of a gauge-changeable truck driven by direct drive motors (DDMs) because they feature high running stability ${ }^{(1)}$. Also, owing to the structural feature that the left and right wheels do not require common axle, the adoption of IRWs in low-floored trams is proceeding ${ }^{(2)}$.

However, unlike the SW, IRWs lack the ability to guide a vehicle along a track, which is known as self-steering. Therefore, it is of concern that a truck with IRWs tends to run moving laterally due to the effect of misalignment between the leading and trailing axles, which is an error generated in the truck assembly. In other words, because of the one-sided running caused by the truck with misaligned axles, it is expected that the wheel flange will be worn by the continuous contact with the side of the rail even on a tangent track ${ }^{(3),(4)}$.

There have been few reports on the effect of the axle misalignment of IRWs. In this paper we aim to relate the wheel lateral force generated by continuous flange contact on a tangent track owing to axle misalignment to that occurring during curve negotiation. In this paper, the wheel lateral force generated by flange contact is calculated quantitatively by

"Received 2 Apr., 2010 (No. T2-08-0543) Eng., Vol.74, No.748, C (2008), pp.2932-2938 (Received 16 June, 2008) [DOI: $10.1299 / j m t l .3 .443]$

Copyright $@ 2010$ by JSME 
numerical simulation using an analytical full vehicle model with IRWs.

\section{Nomenclature}

The main symbols and their values used in this paper are as follows, $m_{w s}$ : Mass of wheelset consisting wheels and axle

$m_{t}$ : Truck frame mass

$I_{w s}$ :Yaw moment of inertia as wheelset

$I_{w y}$ : Moment of inertia of wheel rolling about axle

$I_{t}$ : Yaw moment of inertia of truck frame

$k_{x}$ : Longitudinal support stiffness of axle box

$k_{y}$ : Lateral support stiffness of axle box

$2 a$ : Wheelbase

$2 b_{0}$ : Distance between wheel/rail contact points

$2 b_{1}$ : Lateral distance between axle boxes

$R$ : Curve radius

$F_{1 i j}, F_{1 r i}$ : Longitudinal creep force acting between wheel and rail

$F_{2 l i}, F_{2 r i}:$ Lateral creep force acting between wheel and rail

(Suffixes $l, r$ denote left and right wheels, respectively. Likewise, $i$ denotes wheelset position; 1 for the front and 2 for the rear.)

\section{Angular Misalignment and Lateral Misalignment}

Figure 1 shows a schematic drawing of a truck with axle misalignment, which consists of an angular error and a lateral error. Here, the angular error $\psi_{e}$ is the relative yaw angle between the leading and trailing axles. Namely, $\psi_{e}$ corresponds to the sum of the angular errors of the front and rear axles in the yaw direction, $\psi_{e 1}$ and $\psi_{e 2}$. The relation between $\psi_{e}$, $\psi_{e 1}$ and $\psi_{e 2}$ is given in Eq. (1), taking the counterclockwise direction as positive.

$$
\psi_{e}=\left|\psi_{e 1}-\psi_{e 2}\right|
$$

In practice, instead of the relative yaw angle between the wheelsets, the longitudinal distances between the axle boxes on both left and right sides are measured and the difference between them $\left(\Delta L_{x}=\left|L_{x 1}-L_{x 2}\right|\right)$ is used as the error ${ }^{(5)}$. The relation between $\psi_{e}$ and $\Delta L_{x}$ is given in Eq. (2).

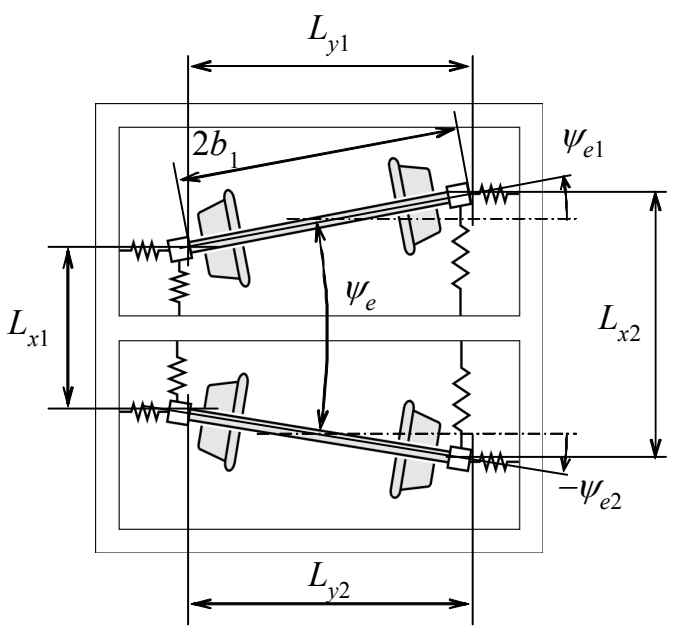

Fig. 1 Truck with axle misalignment 


$$
\psi_{e}=\frac{\Delta L_{x}}{2 b_{1}}
$$

Figure 2 shows the axle misalignment $\psi_{e}$ for the case of $2 b_{1}=1.62 \mathrm{~m}$, considering an electric car used on conventional railway lines. The range of permitted values for $\Delta L_{x}$ is 2 $\mathrm{mm}$ or less ${ }^{(5)}$, and the value of $\psi_{e}$ that corresponds to $\Delta L_{x}=2 \mathrm{~mm}$ is about $1.2 \mathrm{mrad}$. This value is the maximum permitted value in assembly, and it is likely that the value of $\Delta L_{x}$ increases during operation. Considering this increase in the value of $\Delta L_{x}$, the maximum value of $\Delta L_{x}$ considered is increased to $3 \mathrm{mrad}$ in this study.

Then the effect of the lateral error in axle misalignment is investigated similarly. The lateral error is the lateral difference in the locations of the axle boxes. In the assembly of a truck, the lateral distances between the left and right axle boxes are measured, and the difference between them is calculated as $\Delta L_{y}=\left|L_{y 1}-L_{y 2}\right|$. The range of permitted values for $\Delta L_{y}$ is also $2 \mathrm{~mm}$ or less ${ }^{(5)}$, and $\Delta L_{y}=2 \mathrm{~mm}$ is set as the maximum lateral error in this study.

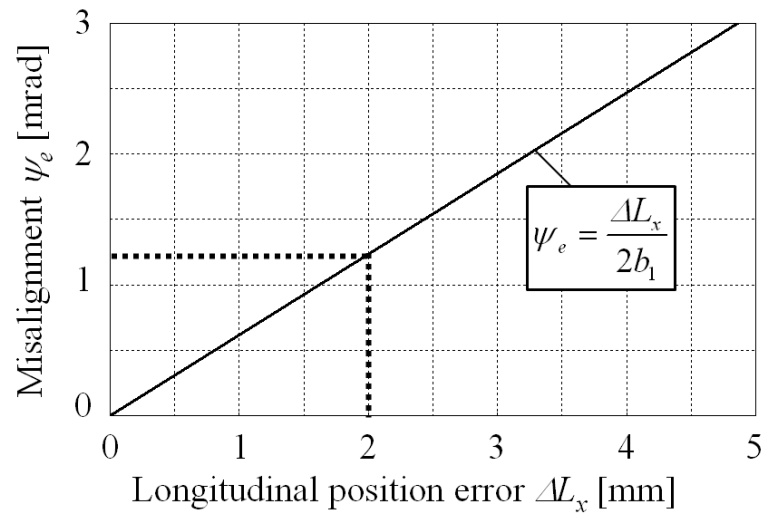

Fig. 2 Relationship between longitudinal position error of axle boxes and axle misalignment

\section{Analytical Model}

\subsection{Model of truck with axle misalignment}

Figure 3 shows the analytical model of a truck with axle misalignment in the yaw direction. Here, creep forces are generated as a result of slippages between the wheel and rail; the longitudinal forces are $F_{1 l i}$ and $F_{1 r i}$, and the lateral creep forces are $F_{2 l i}$ and $F_{2 r i}$. The asymptotic property of creep force toward the frictional force is modeled using the Lévi and Chartet formulation ${ }^{(6)}$.

In this truck model, the angular misalignment $\psi_{e}$ is described as the relative yaw angle between the axles, obtained from the angular errors in the yaw direction $\psi_{e i}$. In the case that the $i$-th axle in the analytical model has yaw angular error $\psi_{e}$, the lengths of the relevant longitudinal springs are adjusted to make the yaw moment equal to zero, while maintaining the angular error. Similarly, the lengths of the lateral springs are also adjusted in the case of a lateral misalignment. Considering the lateral misalignment $y_{e i}$ and yaw angular error $\psi_{e i}$ in each axle, the equations of motion for the truck model are given as follows.

$$
\begin{aligned}
m_{w s} \ddot{y}_{1}-2 k_{y}\left(y_{t}-y_{1}+a \psi_{t}+y_{e 1}\right) & =F_{2 r 1}+F_{2 l 1} \\
m_{w s} \ddot{y}_{2}-2 k_{y}\left(y_{t}-y_{2}-a \psi_{t}+y_{e 2}\right) & =F_{2 r 1}+F_{2 l 1}
\end{aligned}
$$




$$
\begin{gathered}
m_{t} \ddot{y}_{t}+2 k_{y}\left(2 y_{t}-y_{1}-y_{2}+y_{e 1}+y_{e 2}\right)=0 \\
I_{w s} \ddot{\psi}_{1}-2 k_{x} b_{1}^{2}\left(\psi_{t}-\psi_{1}+\psi_{e 1}\right)=b_{0}\left(F_{1 r 1}-F_{1 l 1}\right) \\
I_{w s} \ddot{\psi}_{2}-2 k_{x} b_{1}^{2}\left(\psi_{t}-\psi_{2}+\psi_{e 2}\right)=b_{0}\left(F_{1 r 2}-F_{1 / 2}\right) \\
I_{t} \ddot{\psi}_{t}+2 k_{x} b_{1}^{2}\left(2 \psi_{t}-\psi_{1}-\psi_{2}+\psi_{e 1}+\psi_{e 2}\right)+2 k_{y} a\left(2 a \psi_{t}-y_{1}+y_{2}+y_{e 1}-y_{e 2}\right)=0
\end{gathered}
$$

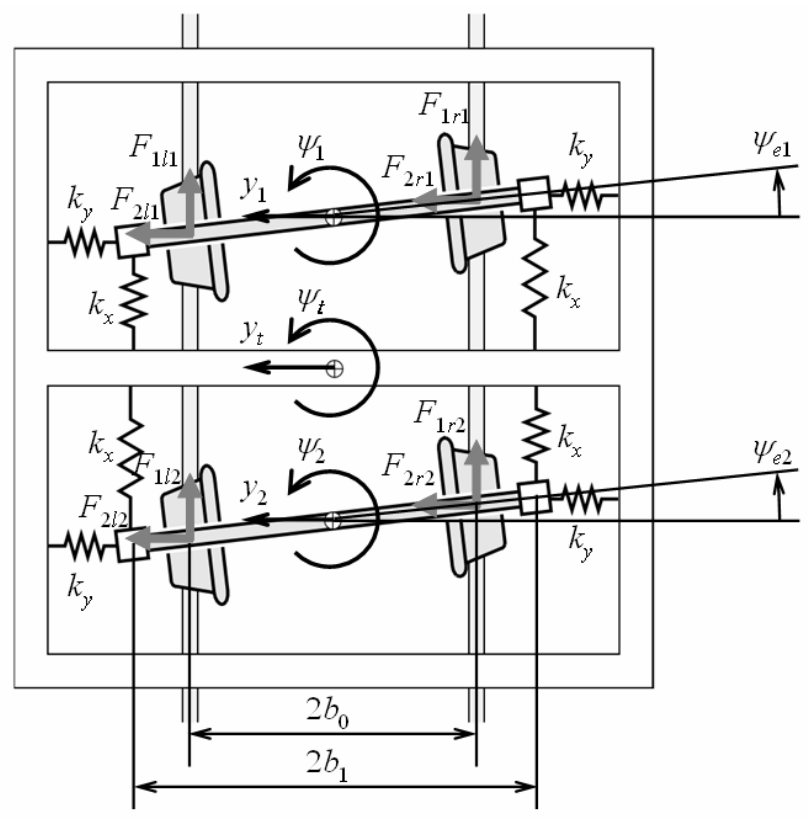

Fig.3 Analytical model of truck with axle misalignment

\subsection{Modeling of axle with independently rotating wheels}

Figure 4 shows the analytical model of an axle with IRWs ${ }^{(7)}$. The right and left wheels, each combined with a DDM, are installed on a non-rotational axle through bearings. Each wheel rotates independently but moves together with the axle in the lateral and yaw directions. Thus, the model has four degrees of freedom in total: lateral motion $y$, yaw angular motion $\psi$, and the rotations of the left and right wheels $\theta_{l}$ and $\theta_{r}$.

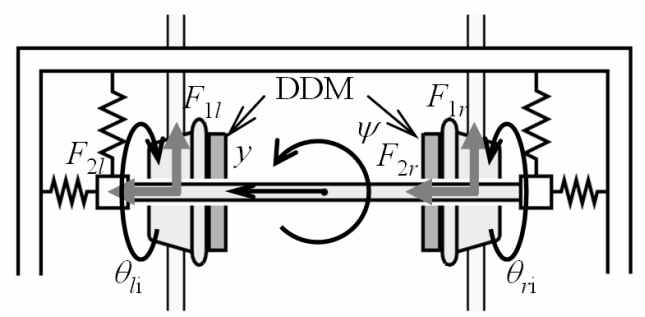

Fig. 4 Analytical model of IRWs

In addition to using Eqs. (3) - (8), the rotations of the left and right wheels, $\theta_{l i}$ and $\theta_{r i}$, are considered for the truck model with two axles by Eqs. (9) and (10), respectively, in which $i=1$ for the front axle and $i=2$ for the rear axle.

$$
I_{w y} \theta_{l i}=-R_{w l i} \cdot r_{l i}+T_{A l i}
$$




$$
I_{w y} \theta_{r i}=-R_{w r i} \cdot r_{r i}+T_{A r i}
$$

Here, $R_{w l i}$ and $R_{w r i}$ are the running resistances acting on each wheel, $r_{l i}$ and $r_{r i}$ are the rolling radii of each wheel, and $T_{A l i}$ and $T_{A r i}$ are the torques generated by each DDM. In addition, in the range of creep force below its saturation with increasing slippage, the longitudinal creep forces can be expressed as $F_{1 l i}=T_{A l i} / r_{l i}$ and $F_{1 r i}=T_{A r i} / r_{r i}$. Then, Eq. (11) becomes applicable for the case that the truck runs at a constant speed.

$$
\sum_{i}\left(F_{1 l i}+F_{1 r i}\right)=\sum_{i}\left(R_{w l i}+R_{w r i}\right)
$$

Thus, it is possible to represent a driving wheel utilizing this IRW model. In Section 4.3 , the effects of the location of the driving wheels will be examined, where $T_{A l 2}=T_{A r 2}=0$ means front-wheel drive and $T_{A l 1}=T_{A r 1}=0$ means rear-wheel drive.

\subsection{Vehicle model}

Among the tram cars with IRWs, vehicles with an articulated body structure exist. In such vehicles each articulated car body is equipped with one truck. It can be assumed for such vehicles that there is little restraint in the yaw direction between the front and rear car bodies. Thus, a lateral model of the vehicle with a car body on one truck ${ }^{(8)}$ is considered in this study. The structure of the vehicle model is based on a 2-axle bogie vehicle, where the solid wheelsets are replaced with IRWs each with a DDM. As shown in Fig. 5, the vehicle model has 13 degrees of freedom of motion in total: lateral motion $y_{b}$ and roll motion $\phi_{b}$ for the car body; lateral motion $y_{t}$, roll motion $\phi_{t}$ and yaw motion $\psi_{t}$ for the truck frame; lateral motion $y_{i}$ and yaw motion $\psi_{i}$ for the axles $(i=1$ for the front axle and $i=2$ for the rear axle); and rotation angles $\theta_{l i}$ and $\theta_{r i}$ for the left and right wheels in each axle, respectively.
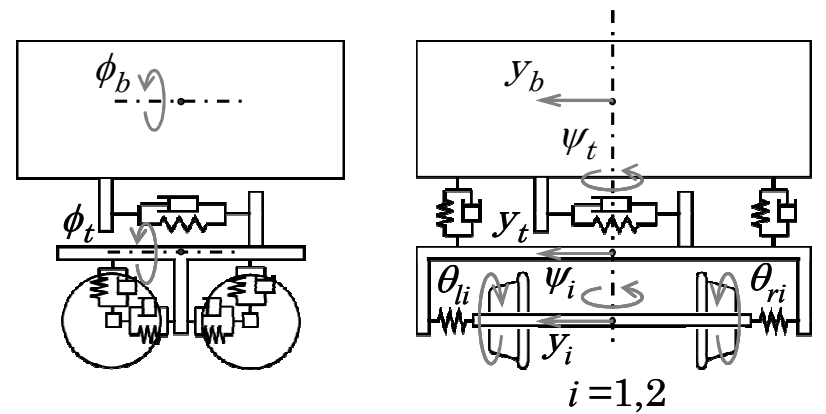

Fig.5 Analytical model of full vehicle

\section{Effects of Misalignment on Wheel Lateral Force}

The effects of angular misalignment $\psi_{e}$ on the running behavior on a tangent section are examined by numerical simulation. Here, the wheel lateral force is used to evaluate the effects quantitatively. The vehicle parameter values used in the following analysis are those corresponding to an electric car on conventional railway lines. The running speed is set to $40 \mathrm{~km} / \mathrm{h}$, which is the maximum allowable speed for tramcars.

\subsection{Behavior of a Vehicle with Angular Misalignment}

In Fig. 6, the behavior on a tangent section is compared between a vehicle with IRWs and one with SWs in terms of the lateral displacements $y_{i}$, yaw angles $\psi_{i}$ and lateral forces $Q_{i}$. Both vehicles have yaw angle errors; $\psi_{e 1}=1.2 \mathrm{mrad}$ for the front axle and $\psi_{e 2}=0$ for the rear axle. Here, $Q_{i}$ is obtained as the sum of the flange reaction force and the lateral creep force on the flanging wheel in each axle; $i=1$ for the front axle and $i=2$ for the rear axle $^{(6)}$. 

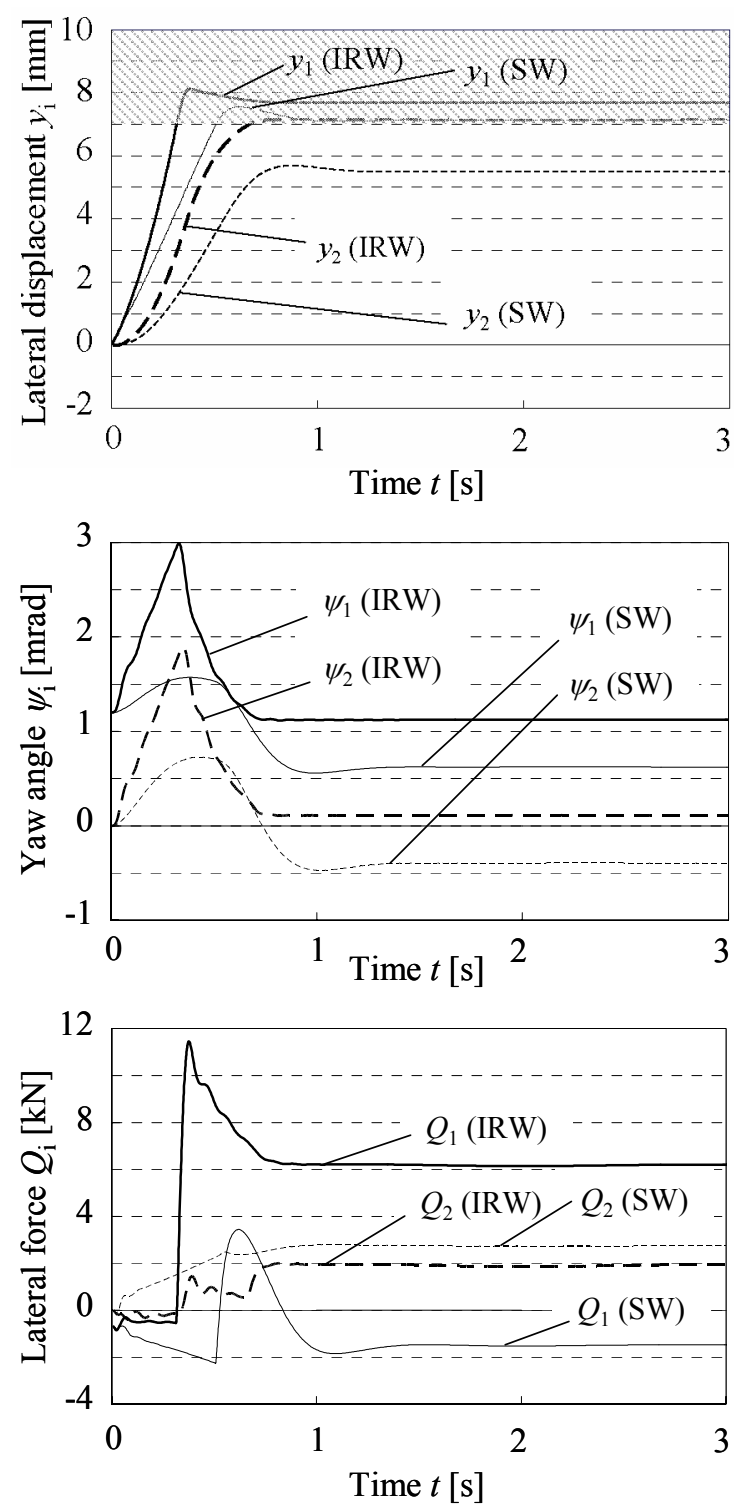

Fig 6 Comparison of behavior between vehicles with IRWs and SWs

$$
\left(\psi_{e 1}=1.2 \mathrm{mrad}\right)
$$

It is shown from Fig. 6 that owing to the axle misalignment, the axles move laterally from the initial value of $y_{i}=0$ and the wheel flange comes in contact with the rail. The clearance between the wheel flange and the rail is $7 \mathrm{~mm}$; thus, values of $y_{i}$ in the hatched area of Fig. 6 indicate flange contact. The IRWs, which have no self-steering ability, make earlier flange contact than the SWs, which have self-steering ability. Under steady behavior with flange contact after about $t=1.5 \mathrm{~s}$, the yaw angle in the front axle of the IRWs, $\psi_{1}$, is almost the same as the misalignment, $\psi_{e}$, and is larger than that for the SWs. The yaw angle $\psi_{1}$ corresponds to the attack angle. Therefore, the absolute value of the wheel lateral force in the front axle, $Q_{1}$, is greater on the IRWs than that acting on the SWs.

As mentioned above, a problem of the truck with IRWs is that one-sided running occurs owing to the yaw angle error, generating a sustained front lateral force even on a tangent track. From Section 4.3, the effects of $\psi_{e}$ are evaluated, focusing on the value of $Q_{1}$ under steady behavior after flange contact. 


\subsection{Behavior of a vehicle with lateral misalignment}

The effects of lateral misalignment on vehicle behavior are examined here. Figure 7 shows a comparison of running behaviors on a tangent section between the vehicle with driving IRWs and that with SWs. Here, the lateral error of the front axle is $y_{e 1}=2 \mathrm{~mm}$ and that of the rear axle is $y_{e 2}=0$ for both vehicles. In Fig. 7, it is shown that the vehicle with IRWs runs straight. In addition, the vehicle with SWs, in which wheelsets are steered by self-steering, becomes stable with increasing time. Also, in the case that the rear axle has lateral error $y_{e 2}=2 \mathrm{~mm}$ and the front axle has lateral error $y_{e 1}=0$, the vehicles exhibit the same behavior as that in the case described in Section 4.1. From these results, it can be concluded that for the range of lateral misalignment under consideration one-sided running does not occur. As a result, setting $y_{e i}$ to be 0 , the effects of $\psi_{e}$ will be focused on in the following analysis.

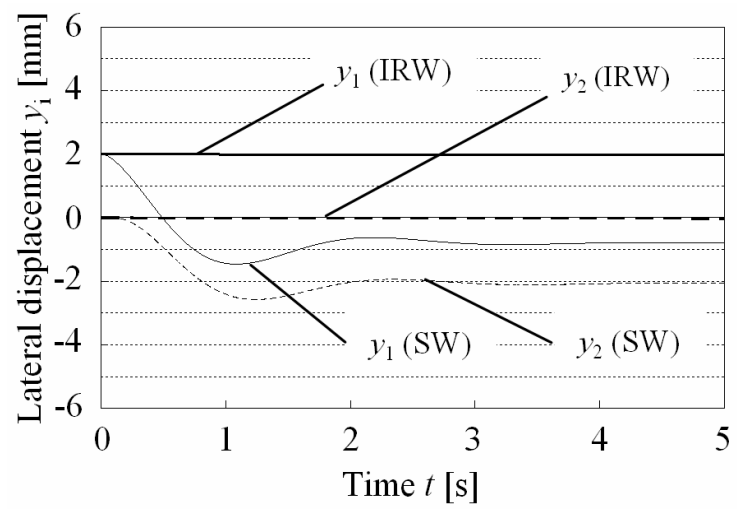

Fig. 7 Comparison of behavior between vehicles with IRWs and SWs $\left(y_{e 1}=2 \mathrm{~mm}\right)$

\subsection{Effect of location of driving wheels}

It is important to examine the effect of the location of driving wheels on the wheel lateral force $Q_{1}$ since the IRWs considered in this study may have a DDM. Figure 8 shows a comparison of $Q_{1}$ on a tangent section for three cases: (A) front-wheel drive, (B) rear-wheel drive and $(\mathrm{C})$ four-wheel drive. Here, the yaw angle error of the front axle $\psi_{e}$ is varied by increments of $0.5 \mathrm{mrad}$ in the range of 0.5 to $3.0 \mathrm{mrad}$. Little difference can be seen in the values of $Q_{1}$ among the three cases.

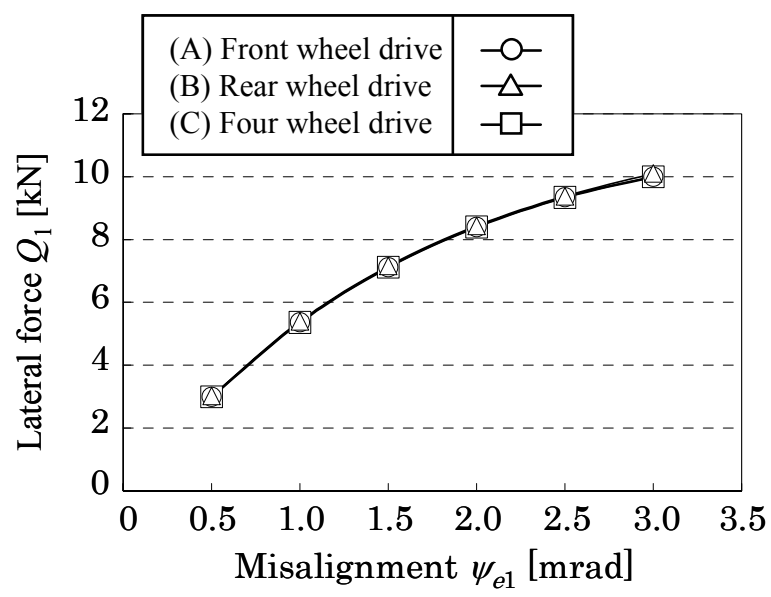

Fig. 8 Comparison of wheel lateral force for different positions of driving wheels (IRWs) 
Focusing on the effect of the increase in $\psi_{e 1}, Q_{1}$ increases with $\psi_{e 1}$, although the rate of increase of $Q_{1}$ decreases gradually. This is due to the property of the lateral creep force; that is, the lateral creep force approaches to the frictional force with the increase of slippage, owing to the increase of the attack angle.

\subsection{Effects of the position of the yaw angle error}

It is supposed that the effect of the angular misalignment $\psi_{e}$ on the wheel lateral force depends on whether that the angular error of the front axle $\psi_{e 1}$ is larger than that of the rear axle $\psi_{e 2}$ or vice versa, even if $\psi_{e}$, which is the relative yaw angle between the front and rear axles, is the same. Thus, the effect of the relative magnitudes of $\psi_{e 1}$ and $\psi_{e 2}$ is examined. In this paper, the following three simplified cases are considered: (D) an error only in the front axle $\left(\psi_{e 1}=\psi_{e}\right),(\mathrm{E})$ an error only in the rear axle $\left(\psi_{e 2}=-\psi_{e}\right)$, and $(\mathrm{F})$ equal errors in the front and rear axles $\left(\psi_{e 1}=\psi_{e} / 2, \psi_{e 2}=-\psi_{e} / 2\right)$. Here, four-wheel drive Case (C) is employed for the driving wheel location.

Figure 9 shows a comparison of $Q_{1}$ occurred on a tangent section for Cases (D), (E) and (F), with $\psi_{e}$ varied by increments of $0.5 \mathrm{mrad}$ in the range of 0.5 to $3.0 \mathrm{mrad}$. It is shown in Fig. 9 that the wheel lateral force on the IRWs is greater in Case (D), in which the error is only in the front axle, than those in Cases (E) and (F). Figure 10 shows the yaw angle of the front axle $\psi_{1}$ with the angular misalignment $\psi_{e}$. It turns out that $\psi_{1}$ in case (D) is greater than those in Cases (E) and (F). This is attributed to the result that for Case (D), in which the yaw angle error is only in the front axle, the value of $Q_{1}$ is slightly larger. However, the difference is so small that it cannot be stated conclusively that the position of yaw angle error significantly affects the wheel lateral force.

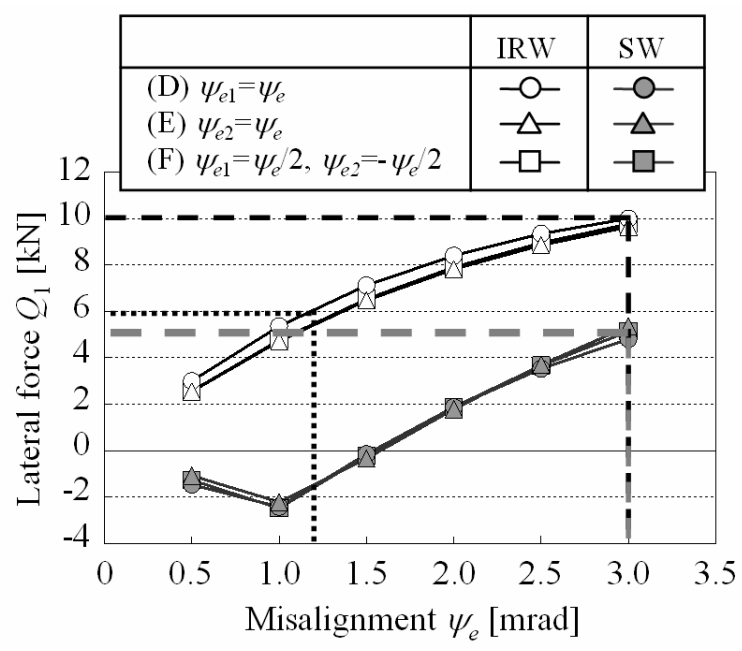

Fig. 9 Comparison of wheel lateral force for different positions of yaw angle error, and between IRWs and SWs

\subsection{Comparison between IRW and SW}

In Fig. 9 described in Section 4.4, the wheel lateral force $Q_{1}$ for the SWs is also shown with $Q_{1}$ for the IRWs. Focusing on the positions of yaw angular error among Cases (D) to (F), the effect of the position on $Q_{1}$ for the SWs is as small as that for the IRWs. In the comparison of $Q_{1}$ acting on the SWs with that acting on the IRWs, the absolute value of the latter is larger than that of the former. Accordingly, in the assumed range of angular misalignment $\psi_{e}$, the wheel lateral force $Q_{1}$ acting on the IRWs is larger than that acting on the SWs, for the same $\psi_{e}$. 


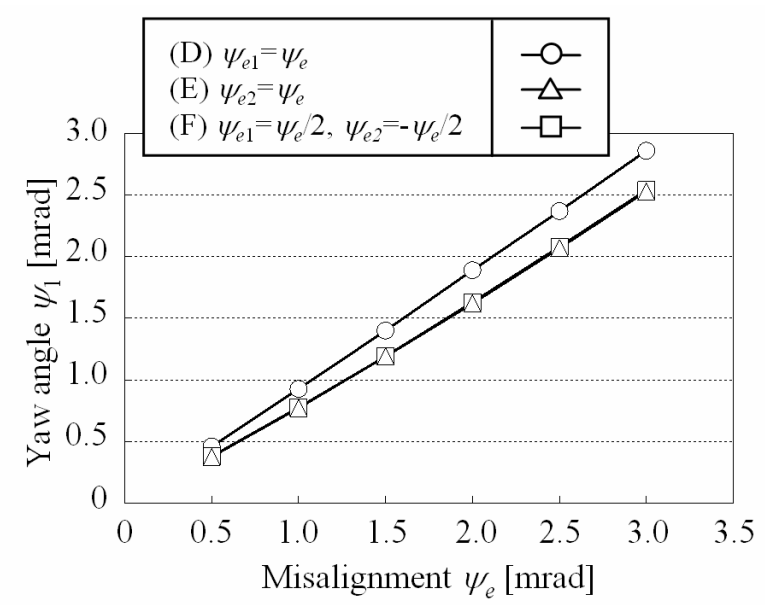

Fig. 10 Comparison of yaw angle of front axle for different positions of yaw angle error (IRWs)

5. Comparison of Running on Tangent Track with Angular Misalignment and Curve Negotiation

A vehicle with an angular misalignment $\psi_{e}$ tends to have sustained flange contact even on a tangent track. Such behavior is similar to that during the curve negotiation of a vehicle without angular misalignment. Thus, to clarify the effect of angular misalignment $\psi_{e}$ on running on a tangent track, the wheel lateral force $Q_{1}$ acting on the front axle is compared with that during curve negotiation.

\subsection{Relation between angular misalignment and curve radius}

Figure 11 shows the geometric relation of a rigid truck in a curved track. Letting $\psi$ be the attack angle, $R$ be the curve radius and $2 a$ be the wheelbase, the relation between them is

$$
\psi=\frac{a}{R} .
$$

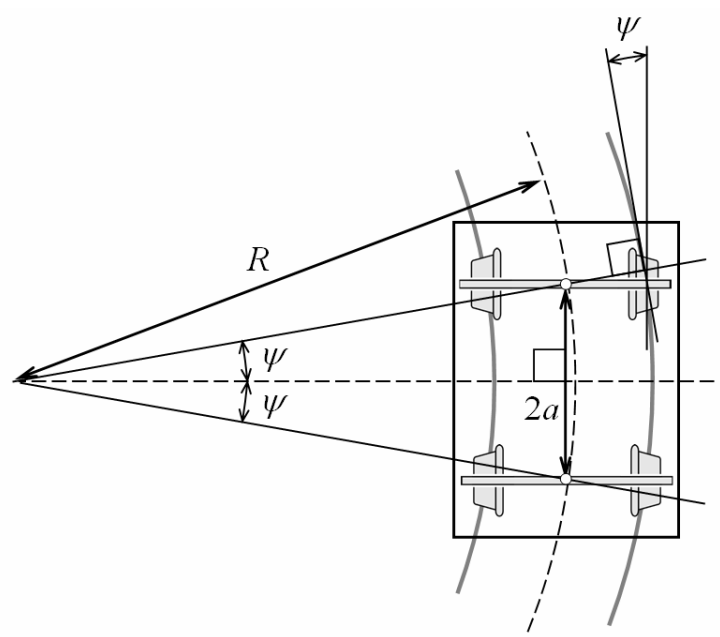

Fig. 11 Rigid two-axle truck negotiating a curve

In addition, the central angle of the arc corresponding to the wheelbase of $2 a$ becomes $2 \psi$ for a curve with radius $R$. Here, the attack angle is $\psi$ on the front axle and $-\psi$ on the rear axle; therefore, it is assumed that the relative yaw angle between the front and rear axles in a curve, corresponds to the angular misalignment $\psi_{e}$ on a tangent track. Namely, $2 \psi=\psi_{e}$. Thus, the relation between $\psi_{e}$ and $R$ is given by 


$$
R=\frac{2 a}{\psi_{e}} .
$$

This relation in the case of $2 a=2.3 \mathrm{~m}$ is shown in Fig. 12. Hence, it is presumed that the maximum permitted error in truck assembly, $\psi_{e}=1.2 \mathrm{mrad}$, corresponds to a curve radius $R$ of about $2000 \mathrm{~m}$.

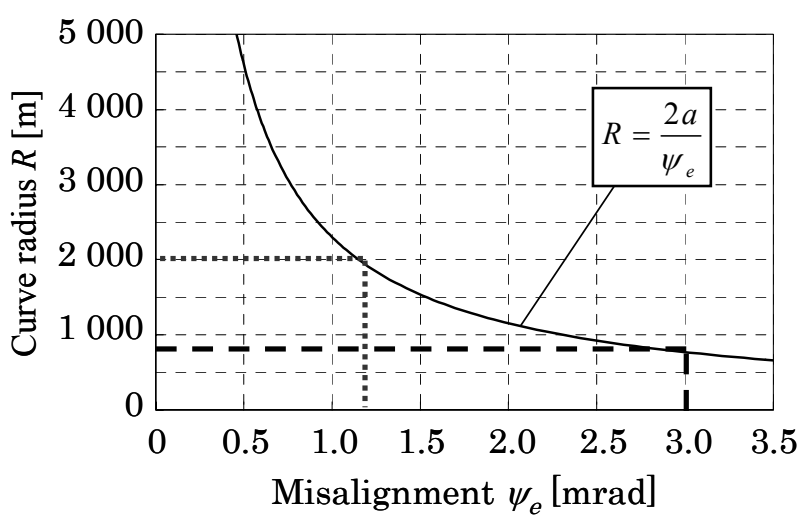

Fig. 12 Relationship between axle misalignment and curve radius

\subsection{Comparison with curve negotiation}

Figure 13 shows the wheel lateral force $Q_{1}$, obtained by numerical simulation, acting on IRWs and SWs in circular curve sections. Here, the angular misalignment is set to $\psi_{e}=0$ and $R$ is varied by increments of $200 \mathrm{~m}$ in the range of 600 to $2400 \mathrm{~m}$.

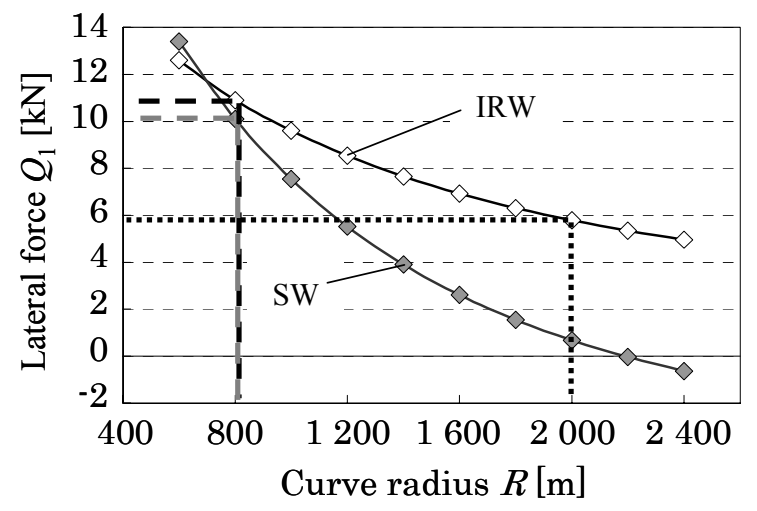

Fig. 13 Comparison of wheel lateral force in curve between vehicles with IRWs and SWs

The curve radius that corresponds to the angular misalignment of $\psi_{e}=1.2 \mathrm{mrad}$ is $R=$ 2000 m, as shown in Fig. 12. Also, from the simulation result shown in Fig. 9, the wheel lateral force $Q_{1}$ acting on the front axle of the IRW vehicle with an angular misalignment of $\psi_{e}=1.2 \mathrm{mrad}$ is about $6 \mathrm{kN}$ on a tangent track. On the other hand, Fig. 13 shows that the value of $Q_{1}$ acting on the IRW vehicle negotiating a curve of $R=2000 \mathrm{~m}$ is also about 6 $\mathrm{kN}$. These results show that the value of $Q_{1}$ generated on a tangent track by an angular misalignment of $\psi_{e}=1.2 \mathrm{mrad}$ is equivalent to that during the negotiation of a curve of radius $R=2000 \mathrm{~m}$.

Next, the wheel lateral force $Q_{1}$ is evaluated for $\psi_{e}=3 \mathrm{mrad}$, which is the maximum angular misalignment under consideration. From Fig. 12, the value of $R$ corresponding to $\psi_{e}$ $=3 \mathrm{mrad}$ is about $800 \mathrm{~m}$. In the running simulation of the IRW vehicle, however, the value of $Q_{1}$ for an angular misalignment of $\psi_{e}=3 \mathrm{mrad}$ is about $10 \mathrm{kN}$, although its value during 
the negotiation of a curve with $R=800 \mathrm{~m}$ is about $11 \mathrm{kN}$. Namely, the wheel lateral force in the latter is slightly larger than that in the former case. This is attributed to the fact that the centrifugal force during curving becomes greater with decreasing $R$. However, the difference is so small that it is possible to consider that the value of $Q_{1}$ generated by the angular misalignment of $\psi_{e}=3 \mathrm{mrad}$ on a tangent track is close that generated during the negotiation of a curve with $R=800 \mathrm{~m}$. Moreover, from the relations between $\psi_{e}$ and $R$ shown in Fig. 12, it is shown from the simulation results in Figs. 9 and 13 that equal wheel lateral forces are generated under both running conditions.

As mentioned above, it can be concluded that for the vehicle with IRWs the wheel lateral force $Q_{1}$ generated on a tangent track by angular misalignment is equivalent to the value generated during the negotiation of a curve of radius $R$ from Eq. (13).

Similarly, the wheel lateral force $Q_{1}$ for the SW vehicle is compared between the conditions of an angular misalignment of $\psi_{e}=3 \mathrm{mrad}$ and the negotiation of a curve with $R$ $=800 \mathrm{~m}$. Figure 9 shows that the value of $Q_{1}$ on the SW vehicle with $\psi_{e}=3 \mathrm{mrad}$ is about 5 $\mathrm{kN}$. However, as shown in Fig. 13, during the negotiation of the corresponding curve with $R$ $=800 \mathrm{~m}, Q_{1}$ increases to about $10 \mathrm{kN}$. In the $\mathrm{SW}$ vehicle, the rear axle moves toward the inner rail with decreasing curve radius, and the yaw moment generated by the longitudinal creep force pushes the front axle onto the outer rail through the truck frame. This causes the larger value for $Q_{1}$ for the $\mathrm{SW}$ vehicle ${ }^{(9)}$.

As stated above, it is shown for the IRW vehicle that flange contact due to angular misalignment $\psi_{e}$ is sustained even on a tangent track, and an equivalent wheel lateral to that during the negotiation of a curve with the corresponding radius force is continuously generated, resulting in the rapid wear of the wheel flange.

\section{Conclusions}

An analytical model of a vehicle with axle misalignment was developed and numerical simulations were carried out to examine the effects of the axle misalignment on the running behavior of a vehicle with IRWs on a tangent track. The obtained results are as follows.

(1) The vehicle with IRWs has no self-steering ability. Accordingly, even a slight angular misalignment causes the vehicle to move laterally and one-sided running to occur on a tangent track. This results in sustained flange contact on the front axle and leads to a greater wheel lateral force.

(2) Without any angular misalignment, flange contact due to one-sided running does not occur even if a lateral misalignment exists between the front and rear axles. Furthermore, on a vehicle with angular misalignment, the effects of the location of the driving wheels and the position of yaw angle error on the wheel lateral force are small.

(3) With increasing angular misalignment, the wheel lateral force of the IRWs due to flange contact becomes greater. However, the rate of increase in the wheel lateral force decreases gradually, since the creep force owing to the increase in attack angle approaches to the frictional force.

(4) In the range of angular misalignment considered, the wheel lateral force acting on the IRW vehicle is larger than that on the SW vehicle for the same angular misalignment.

(5) On a vehicle with IRWs, the wheel lateral force caused by angular misalignment is equivalent to that generated during the negotiation of a curve with the corresponding radius $R$, given by Eq. (13).

As a result, the effects of axle misalignment are clarified quantitatively from the viewpoint of the wheel lateral force acting between the wheel flange and rail. The reduction of continuous flange contact due to axle misalignment in IRW vehicles is an issue to be addressed in the future. Finally, the authors would like to thank Professor J. Piotrowski of Warsaw University of Technology for useful suggestions and discussions, which provided the motivation for this study. 


\section{Appendix 1}

Creep forces $F_{j}$ acting on the wheel tread are calculated with the exponent $m=1.5$,

$$
F_{j} / \mu P=\left[1+\left(f_{K j} v_{j} / \mu P\right)^{-m}\right]^{-1 / m} \cdot\left(v_{j} / v\right)
$$

where $j=1$ represent to be longitudinal and $j=2$ to be lateral. $P$ denotes wheel load, $\mu$ creep coefficient, $f_{K j}$ Kalker's creep coefficient, $v_{j}$ creepage and $v=\sqrt{v_{1}^{2}+v_{2}^{2}}$ resultant creepage.

When a wheelset tends to move laterally exceeding the movable clearance $\delta$ between wheel flange and rail on either side, rail reaction force $F_{R}$ and flange frictional force acting in the forward direction $F_{F}$ are generated,

$$
\left.\begin{array}{c}
F_{R}=-k_{R}\left(y_{i}-\delta\right) \quad: y_{i}>\delta \\
F_{R}=k_{R}\left(y_{i}+\delta\right) \quad: y_{i}<-\delta
\end{array}\right\}
$$

where $y_{i}$ denotes lateral displacement of wheelset $(i=1$ for the front axle and $i=2$ for the rear axle), $k_{R}$ effective lateral stiffness of rail and $\alpha$ flange angle.

The following equation represents the running resistance $R_{E C}$ obtained experimentally for a train running on a horizontal tangent track ${ }^{(10)}$.

$$
R_{E C}=(16.2+0.242 \mathrm{~V}) M+0.275 \mathrm{~V}^{2}
$$

Here, $V$ is the running speed $[\mathrm{km} / \mathrm{h}]$ and $M$ is the gross vehicle mass [t]. Equation (17) describes the total resistance to which the vehicle is subject, then the resistance per wheel is given as

$$
R_{s w}=R_{E C} / 8 .
$$

Since the increase in resistance caused by the contact between the wheel flange and rail is not included in Eq. (11), the resistance due to flange contact is considered separately. Once flange contact occurs, the flange friction force $\mu_{F} F_{F}$ acts in the direction opposite to wheel rotation. Here, $\mu_{F}$ is the friction coefficient and $F_{F}$ is the flange reaction force. Denoting the radius at the point of flange contact by $r_{F}$ and the rolling radius by $r$, the resistance $R_{F}$ due to flange contact is obtained by the following expression for moment equilibrium about the contact point on the tread:

$$
R_{F}=\frac{\mu_{F} F_{F}\left(r_{F}-r\right)}{r}
$$

Thus, the sum of $R_{s w}$ and $R_{F}$ is the running resistance $R_{w}$ acting on the wheel. Here, in the IRW vehicle under consideration, the increase in resistance $R_{F}$ due to flange contact is compensated by the output of the DDM on the flanging wheel.

\section{Appendix 2}

The main symbols and their values used in this paper are as follows:

Mass of wheelset consisting wheels and axle: $m_{w s}=2.5 \mathrm{t}$

Truck frame mass: $m_{t}=3.3 \mathrm{t}$

Yaw moment of inertia as wheelset: $I_{w s}=0.87 \mathrm{t} \cdot \mathrm{m}^{2}$

Moment of inertia of wheel rolling about axle: $\quad I_{w y}=0.09 \mathrm{t} \cdot \mathrm{m}^{2}$

Yaw moment of inertia of truck frame: $I_{t}=2.0 \mathrm{t} \cdot \mathrm{m}^{2}$

Longitudinal support stiffness of axle box: $k_{x}=6000 \mathrm{kN} / \mathrm{m}$ 
Lateral support stiffness of axle box: $k_{y}=6000 \mathrm{kN} / \mathrm{m}$

Wheelbase: $2 a=2.3 \mathrm{~m}$

Distance between wheel/rail contact points: $2 b_{0}=1.12 \mathrm{~m}$

Lateral distance between axle boxes: $2 b_{1}=1.62 \mathrm{~m}$

Curve radius: $R$ (variable)

Longitudinal creep force acting between wheel and rail: $F_{1 l i}, F_{1 r i}$ (variable)

Lateral creep force acting between wheel and rail: $\quad F_{2 l i}, F_{2 r i}$ (variable)

[Suffixes $l, r$ denote left and right wheels, respectively. Likewise, $i$ denotes wheelset position; 1 for the front and 2 for the rear.]

Other parameter values used in the calculation:

Car-body mass $(=13 \mathrm{t})$

Roll radius of inertia of car-body $(=1.5 \mathrm{~m})$

Yaw radius of inertia of truck frame $(=0.78 \mathrm{~m})$

Roll radius of inertia of truck frame $(=0.53 \mathrm{~m})$

Yaw radius of inertia of wheelset $(=0.59 \mathrm{~m})$

Roll radius of inertia of wheel $(=0.30 \mathrm{~m})$

Longitudinal stiffness of air spring $(=131 \mathrm{kN} / \mathrm{m})$

Lateral stiffness of air spring $(=131 \mathrm{kN} / \mathrm{m})$

Vertical stiffness of air spring $(=359 \mathrm{kN} / \mathrm{m})$

Vertical damping coefficient of air spring $(=16.9 \mathrm{kN} \cdot \mathrm{s} / \mathrm{m})$

Damping coefficient of lateral damper $(=58.8 \mathrm{kN} \cdot \mathrm{s} / \mathrm{m})$

Damping coefficient of anti-yaw damper $(=58.8 \mathrm{kN} \cdot \mathrm{s} / \mathrm{m})$

Vertical stiffness of axle spring $(=2000 \mathrm{kN} / \mathrm{m})$

Damping coefficient of axle damper $(=80 \mathrm{kN} \cdot \mathrm{s} / \mathrm{m})$

Lateral distance between air springs $(=1.7 \mathrm{~m})$

Height of center of gravity of car-body from air spring center $(=0.467 \mathrm{~m})$

Height of air spring center from center of gravity of truck frame $(=0.32 \mathrm{~m})$

Height of center of gravity of truck frame from axle center $(=0.06 \mathrm{~m})$

Nominal rolling radius of wheel $(=0.43 \mathrm{~m})$

Conicity of wheel tread $(=1 / 20)$

Friction coefficient between wheel tread and rail $(=0.3)$

Friction coefficient between wheel flange and rail $(=0.24)$

\section{References}

(1) Tokuda, N. and Ishige, M., "High speed performance and construction of independent wheel system bogie with DDM", RTRI report, Vol.22, No.2 (2008), pp.5-10.

(2) Japan Society of Rolling Stock and Machinery ed., "Research on Development of New Light Rail Vehicles being Gentle with People" (in Japanese), Interim Report of JRMA, (1997), pp.77-84.

(3) Griffin, T., "Center Truck Performance on Low-Floor Light Rail Vehicles", TCRP Report, 114 (2006), p.30.

(4) Rail Accident Investigation Branch, "Derailment at Birmingham Snow Hill, Midland Metro: 29 January 2007", Rail Accident Report, (2007), p.16, Department for Transport.

(5) Rolling Stock Department ed., Maintenance and Repair of Rolling Stocks (1) (in Japanese), (1977), pp. 602-626, Japanese National Railways.

(6) The Japan Society of Mechanical Engineers ed., Dynamics of railway vehicles (in Japanese), (1994), pp.20-29, Denkisha-Kenkyukai.

(7) Obata, R., Tanifuji, K., Soma, H. and Masuda, T., "Curving Performance of a Rail Vehicle with Independently Rotating Wheels by Torque Difference Control, Transactions of JSME, series $C$, Vol.72, No.716 (2006), pp.1064-1070.

(8) Yoneyama, A., Tanifuji, K., Soma, H. and Masuda, T., "Curving Performance of a Vehicle 
with Independently Rotating Wheels Driven by One Wheel", Proc. of the 2nd Saitama Block Meeting of JSME, No.060-5 (2006), pp.53-54.

(9) Suda, Y. et al., "Steering Ability of Self-Steering Truck with Independently Rotating Wheels in Trailing Axle", Transactions of JSME, Series C, Vol.64, No.628 (1998), pp.4764-4769.

(10) Maruyama, H. et al., Railroad Engineering for Mechanical Engineer (in Japanese), (1984), pp.28-30, Maruzen. 